\title{
COVID-19 pneumonia-ultrasound, radiographic, and computed tomography findings: a comprehensive pictorial essay
}

\author{
Michaela Cellina $^{1}$ (D) $\cdot$ Carlo Martinenghi $^{2} \cdot$ Pietro Marino $^{3} \cdot$ Giancarlo Oliva $^{1}$ \\ Received: 10 December 2020 / Accepted: 19 January 2021 / Published online: 30 January 2021 \\ (C) American Society of Emergency Radiology 2021
}

\begin{abstract}
Ultrasound, chest X-ray, and computed tomography (CT) have been used with excellent results in diagnosis, first assessment, and follow-up of COVID-19 confirmed and suspected patients. Ultrasound and chest X-ray have the advantages of the wide availability and acquisition at the patient's bed; CT showed high sensitivity in COVID-19 diagnosis. Ground-glass opacities and consolidation are the main CT and X-ray features; the distribution of lung abnormalities is typically bilateral and peripheral. Less typical findings, including pleural effusion, mediastinal lymphadenopathies, the bubble air sign, and cavitation, can also be visible on chest CT. Radiologists should be aware of the advantages and limitations of the available imaging techniques and of the different pulmonary aspects of COVID-19 infection.
\end{abstract}

Keywords Pneumonia, viral · Coronavirus · COVID-19 · Severe acute respiratory syndrome coronavirus $2 \cdot$ Tomography, spiral computed

\section{Introduction}

In December 2019, the outbreak of epidemical pneumonia, of initially unidentified origin, occurred in Wuhan, Hubei Province, China [1]. Its cause was identified in a new virus, the "2019 novel coronavirus (2019-nCoV)" or "severe acute respiratory syndrome (SARS)-CoV-2," which was subsequently named coronavirus disease (COVID-19) on January 9, $2020[1,2]$. As of November 21, 2020, a total of 57,274,018 confirmed cases and 1,368,000 deaths have been reported [3].

COVID-19 typically presents with systemic and/or respiratory manifestations $[4,5]$. Symptoms and signs are nonspecific. The absence of specific therapies and the limited percentage of the population currently vaccinated make crucial the early detection of disease, with the isolation of the infected subjects from the healthy population.

Michaela Cellina

michaela.cellina@asst-fbf-sacco.it

1 Department of Radiology, ASST Fatebenefratelli Sacco, P.zza Principessa Clotilde, 3, 20121 Milan, Italy

2 Department of Radiology, San Raffaele Hospital, via Olgettina 60, 20123 Milan, Italy

3 Department of Emergency Medicine, ASST Fatebenefratelli Sacco, P.zza Principessa Clotilde, 3, 20121 Milan, Italy
The gold standard for diagnosis is the reverse transcriptionpolymerase chain reaction (RT-PCR) on throat swab; however, the results of this test are not immediately available, and some patients can show false-negative results at the onset of the symptoms, depending on the quality of the sample and on the rate of viral replication in the upper respiratory tract [4].

Chest imaging can help the diagnosis and management of COVID-19 confirmed and suspected patients.

The threshold and the type of imaging in patients with suspected/confirmed COVID-19 pneumonia is highly variable and depends on local resources, clinical scenarios, and national recommendations [6].

The aim of this pictorial essay is to show the features of COVID-19 pneumonia in all the available imaging techniques, chest ultrasound (US), X-ray, and computed tomography (CT).

\section{Imaging indications}

Indications to chest imaging execution in suspected COVID19 infection are still unclear.

A multinational consensus statement from the Fleischner Society provided some main and additional recommendations about the use of chest imaging [7], stating that imaging is not indicated as a screening test in asymptomatic subjects and in patients with mild clinical symptoms unless there are at risk for 
Fig. 1 Chest US (a) of a COVID19 patient showing more than $3 \mathrm{~B}$ lines (arrows) visible as vertical hyperechoic artifacts originating from the pleural line. Posterobasal chest US scan (b) of a COVID-19 patient showing a peripheral lung consolidation (arrows)

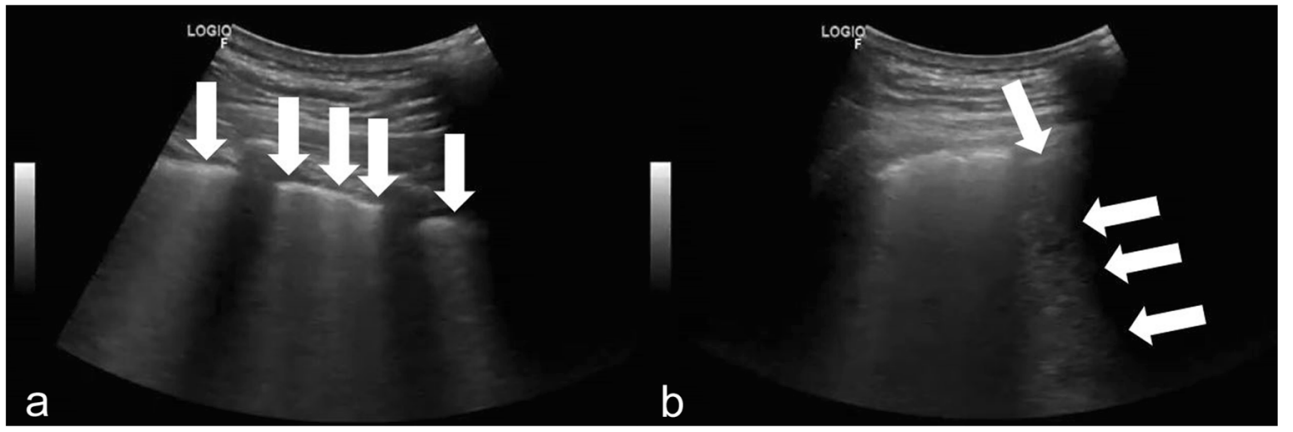

disease progression and that imaging is indicated in suspected cases of COVID-19 with moderate to severe features and in patients with functional impairment after recovery to differentiate between expected abnormalities following COVID-19 infection and/or mechanical ventilation, versus a different and potentially treatable process [2].

Due to its high sensitivity [8], chest CT is widely used as a first-line imaging technique in suspected cases of COVID-19 who present with moderate-severe clinical features and high pretest probability environment $[2,8]$.

The 5th version diagnosis and treatment of COVID-19 issued by the National Health Commission of the People's Republic of China considered CT as a major modality for diagnosis, even before receiving the RT-PCR tests, and propose CT as a useful test for relieving quickly difficult situations in the context where a huge population is waiting for the RT-PCR test, due to the lack of kits, delay waiting for the results [9].

\section{US}

Lung US has been proposed as a potential triage and diagnostic tool in suspected or confirmed COVID-19 patients, due to its wide availability, non-invasiveness, and execution at the bedside. This technique has also the advantage that it can be performed by clinicians who are not radiologists and is a useful tool in monitoring ventilated or intubated COVID-19 patients in the intensive care units (ICU) [10]. The main US finding in COVID-19 pneumonia consists of B lines, which are vertical hyperechoic artifacts departing from the pleura and directing in-depth, representing thickened peripheral interlobular septa.

The visibility of less than $3 \mathrm{~B}$ lines on a scan is considered normal; instead, the evidence of a greater number of $\mathrm{B}$ lines is pathological (Fig. 1), and their number correlates with disease severity $[10,11]$.

The presence of an abnormal number of $\mathrm{B}$ lines is characteristic of different interstitial syndromes, such as pulmonary edema, interstitial pneumonia, and pulmonary fibrosis, and should be always correlated with the patient's current symptoms, clinical presentation and medical history, as well with the epidemiological scenario.

US patterns vary from focal to diffuse lung involvement. In the most serious cases, the B lines are diffuse and confluent, resulting in a single hyperechoic image identified as "white lung." Subpleural consolidations can be identified (Fig. 1b).

The US does not provide a complete overview of parenchyma, as the assessment of the deep lung portions is limited, and this is the main limitation of the technique and is strongly operator-dependent.

\section{Chest X-ray}

Chest X-ray (CXR) is typically the first-line imaging modality for patients with suspected COVID-19 infection. It demonstrated limited sensitivity in mild or early COVID-
Fig. 2 Chest X-rays of two confirmed cases of COVID-19 infection, showing the bilateral presence of GGO (frames). In (a), the distribution is mainly peripheral, whereas in (b), the abnormalities have both peripheral and central distribution in the right lung. In the upper left field (b), some small consolidations are also present

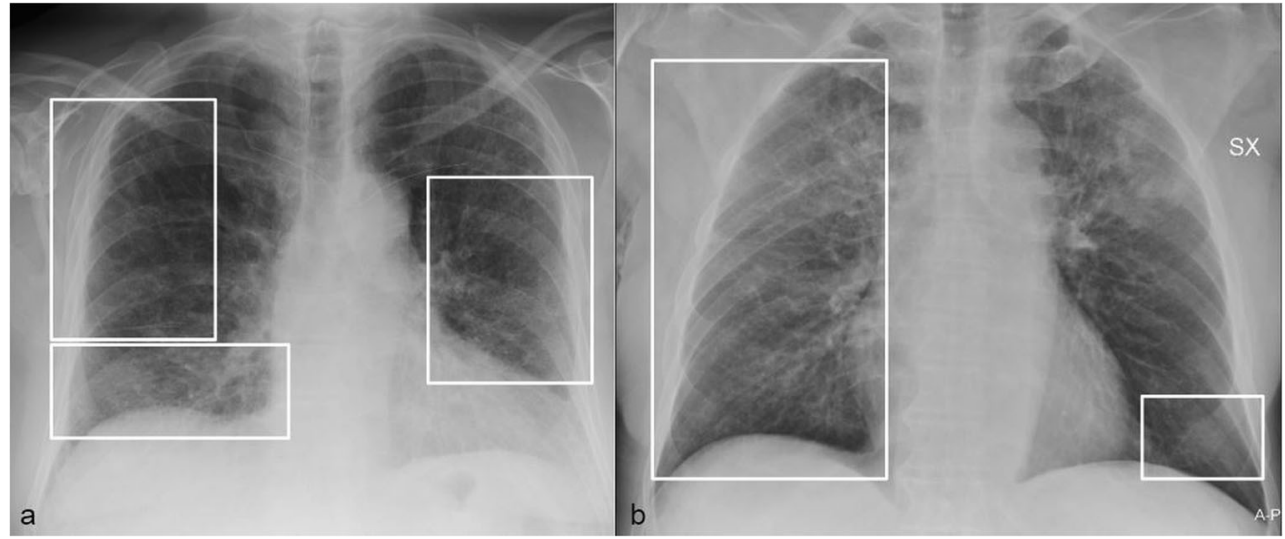


Fig. 3 Examples of consolidations (frames) in two confirmed cases of COVID-19 infection. In (a), the chest radiograph of a 65-year-old man with cough and fever for 7 days, showing bilateral patchy consolidations. In (b), the chest radiography of a 63-year-old man with cough and fever from 12 days, admitted to the intensive care unit showing extensive bilateral consolidation with a prevalent pezones ripheral location in the lower lung
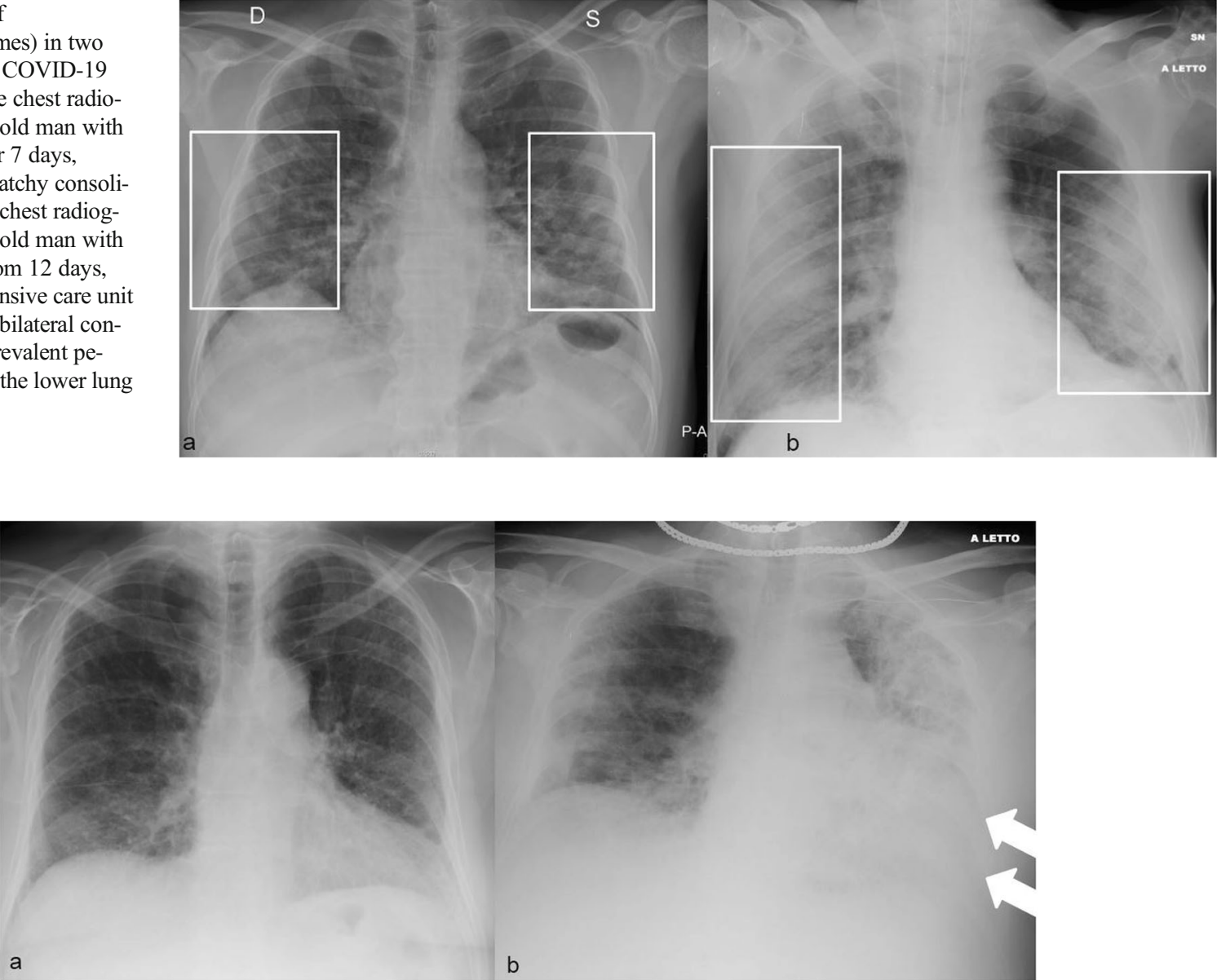

Fig. 4 Changes in lung abnormalities during the disease. Chest X-ray of a 58-year-old man executed on March 18 (a) and on March 30 (b). The first $\mathrm{X}$-ray was acquired at 2 days from the onset of the symptoms and showed bilateral mainly peripheral GGO, and small patchy consolidation in the

19 infection [12], but can be useful for assessing alternative diagnoses, and has a pivotal role in quantifying the severity, monitoring the progression of COVID-19 pneumonia, and evaluating potential complications, as bacterial superinfection, and pneumothorax. left upper field. X-ray following at 12 days showed reduced expansion of the pulmonary fields, with the worsening of the abnormalities and evidence of bilateral consolidation and left pleural effusion (white arrows)

The American College of Radiology advises deploying portable radiography machines in the departments dedicated to the acceptance and treatment of suspected or positive COVID-19 patients, to perform chest X-Rays when a lung evaluation is medically needed [13].
Fig. 5 Chest X-ray (a) and coronal reconstruction of the unenhanced $\mathrm{CT}$ of a 58-year-old COVID-19 male patient admitted to the ICU showing bilateral diffuse airspace opacities and the presence of oval areas of hyperlucency corresponding at $\mathrm{CT}$ to cavitation (frames)
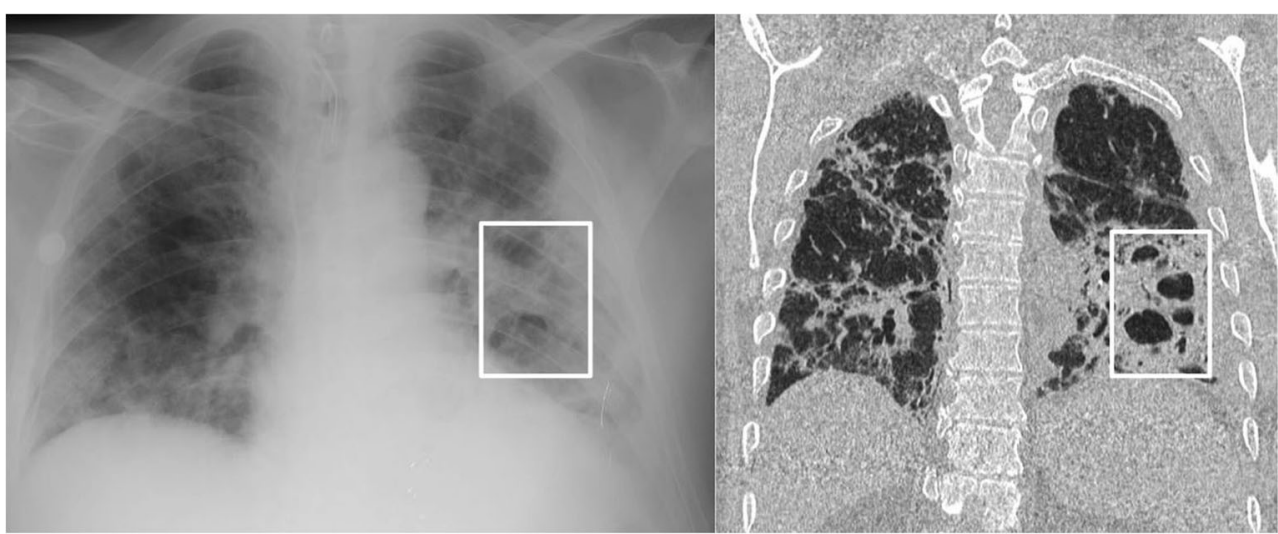


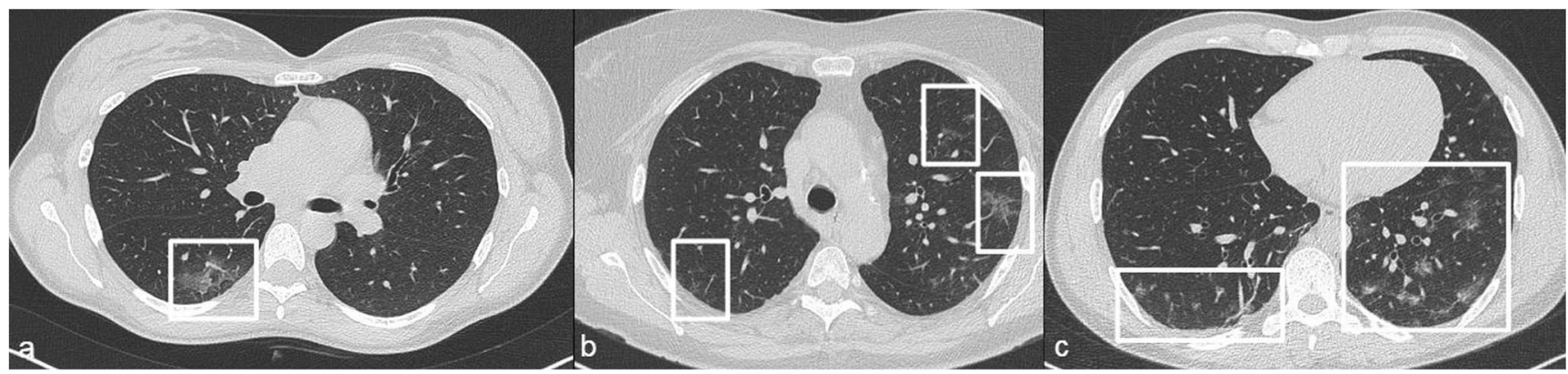

Fig. 6 Unenhanced chest CT scans showing some examples of GGO (frames) in COVID-19 patients. In (a), the abnormality is in the right lower lobe posteriorly; in (b), GGO are bilaterally recognizable, with peripheral and peri-broncho-vascular distribution. In (c), the GGO are patchy, with bilateral involvement of the lower lobes

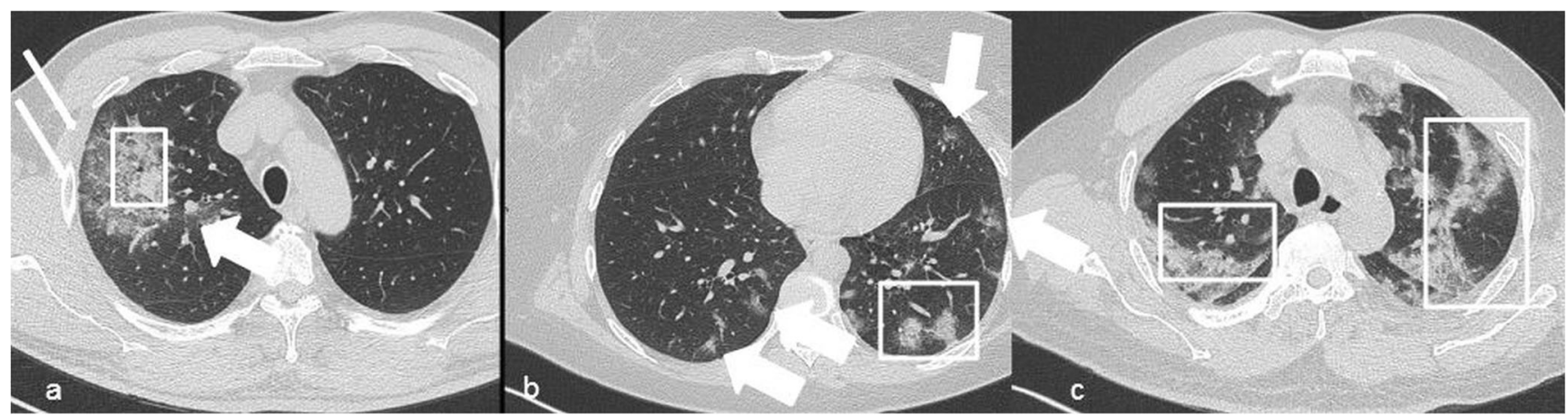

Fig. 7 Examples of lung consolidations (frames) in COVID-19 patients. In (a), the consolidation is associated with GGO (white arrow) and crazy paving pattern (thin arrows); in (b), the consolidations are patchy, with

Chest radiography's main advantages are the wide availability, fast execution, low cost, and acquisition at the patient's bed [14].

Chest radiographs may be normal in early/mild disease, with a reported baseline sensitivity of 69\% [12]. The most frequent findings are ground-glass opacities (GGO) (Fig. 2) and consolidation $[12,15-17]$.

As opposed to community-acquired bacterial pneumonia which tends to be unilateral and involving a single lobe, consolidations in COVID-19 pneumonia tend to be multifocal, patchy, or confluent (Fig. 3). The distribution of chest abnormalities is most often bilateral, peripheral, and lower zone predominant [12-16].

Lung opacities may rapidly evolve into a diffuse coalescent or consolidative pattern within 1-3 weeks of symptoms onset. Findings are most extensive about 10-12 days after symptom onset [12] (Fig. 4).

Pleural effusion is rare (3\%) [12] (Fig. 7b) and seems to be a sign of severe disease [18]. Lung cavitation is a rare finding in COVID-19 patients [19] (Fig. 5).

Pneumothorax is also rather uncommon [20]; it seems to be related to inflammation-induced pulmonary parenchymal injury and necrosis with the development of air leaks into the pleural cavity, a mechanism like that noted in patients during the severe acute respiratory syndrome (SARS) outbreak in 2003 [21]. peripheral posterior location; patchy GGO are also present (arrows). In (c), the consolidations are bilateral and confluent

Diffuse airspace opacities in patients can be seen in severe cases, complicated by acute respiratory distress syndrome (ARDS); when lung disease involves the majority of the pulmonary parenchyma, patients are typically hypoxic and require intubation with mechanical intubation.

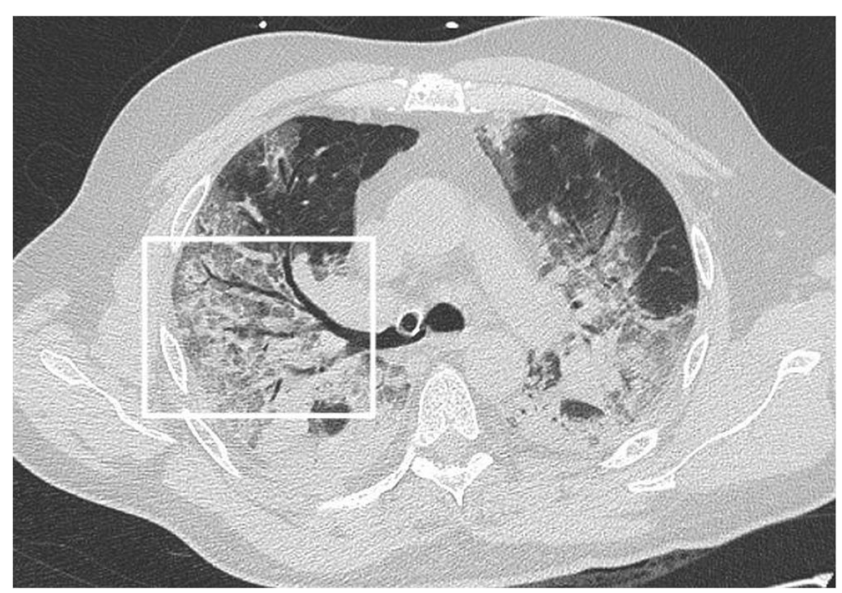

Fig. 8 Unhenanced chest CT of 65-year-old COVID-19 patient admitted to ICU. The tracheal cannula has been wrongly positioned in the right bronchus. Bilateral evidence of extensive airspace abnormalities, with peripheral posterior consolidations and crazy paving pattern and concomitant air bronchogram sign (frame) 
Fig. 9 Examples of reticular patterns in COVID-19 male patients (a 53-year-old, b 63-yearold) showing reticular pattern (frames) with the bilateral peripheral distribution
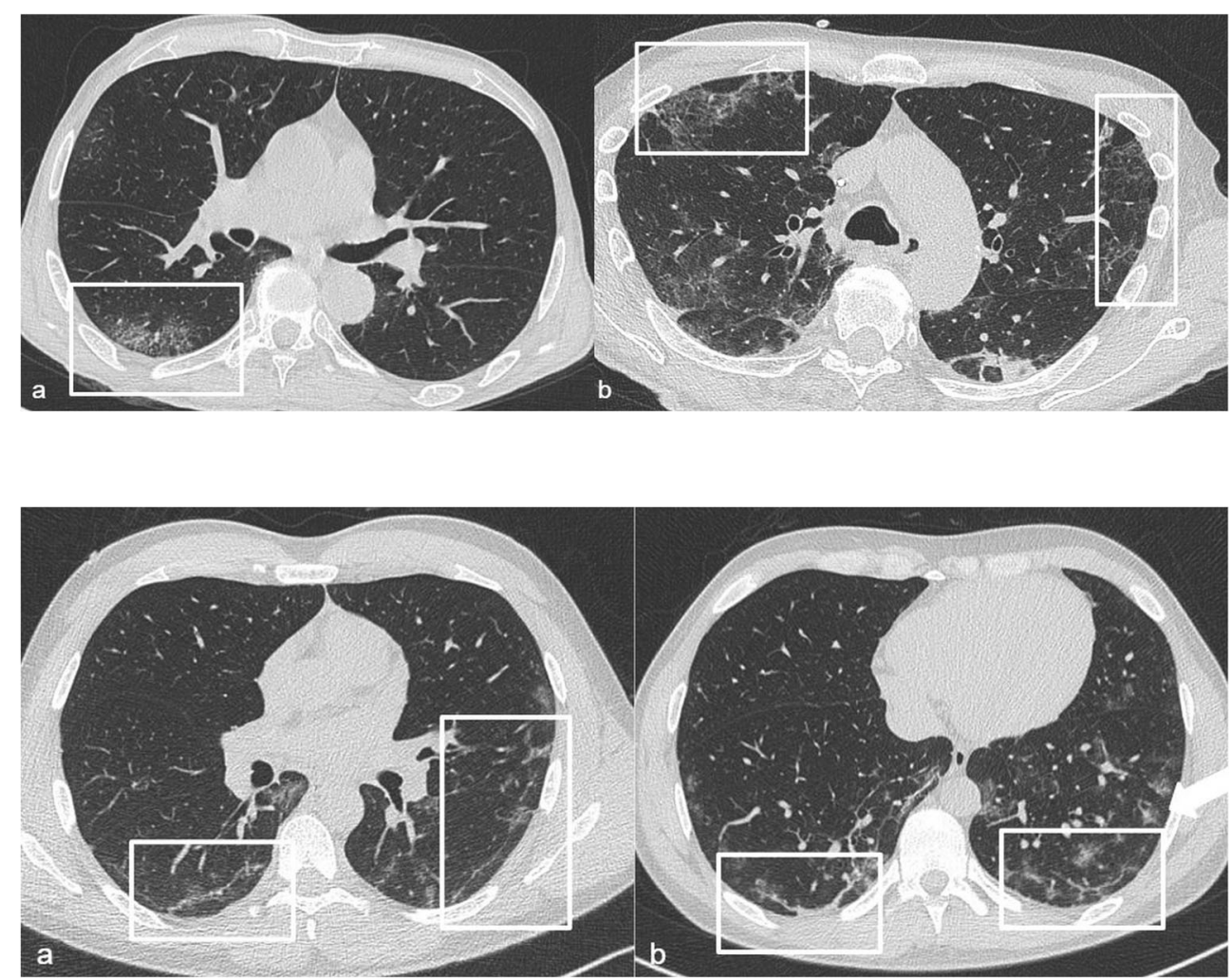

Fig. 10 Chest CT of a 48-yearold patient showing bilateral peripheral linear opacities with course parallel to the pleural surface (frames). A small nodule with peripheral GGO, resulting in a halo sign is present in the lower left lobe (white arrow)

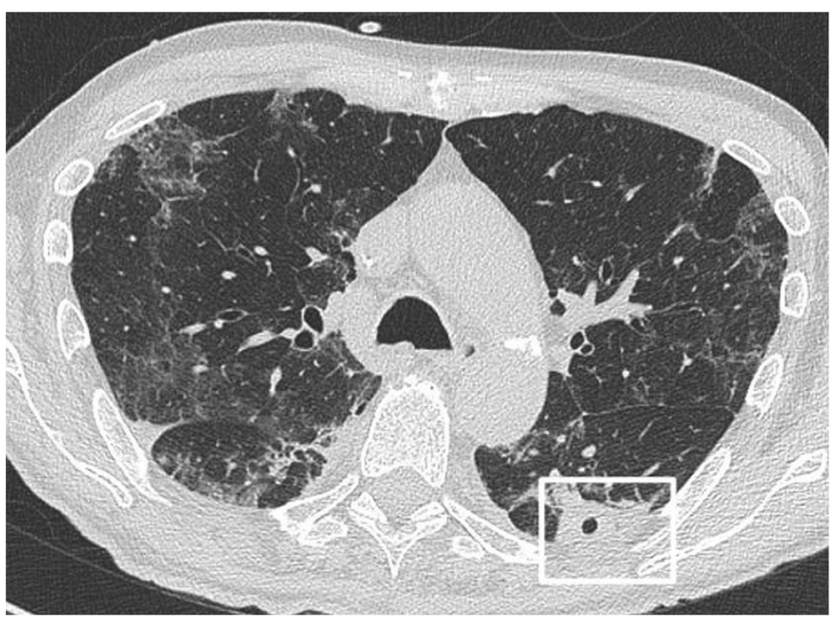

Fig. 11 Chest CT of a 63-year-old COVID-19 patient. In the left lower lobe, posteriorly, a small air-filled cavity in the context of lung consolidation is present (frame). This is the "air bubble sign." Areas of the reticular pattern are bilaterally recognizable

\section{Chest CT}

According to the American College of Radiology recommendations, chest CT should not be used as a screening tool for COVID19; CT should be used sparingly and reserved for hospitalized, symptomatic patients with specific clinical indications for CT [13].

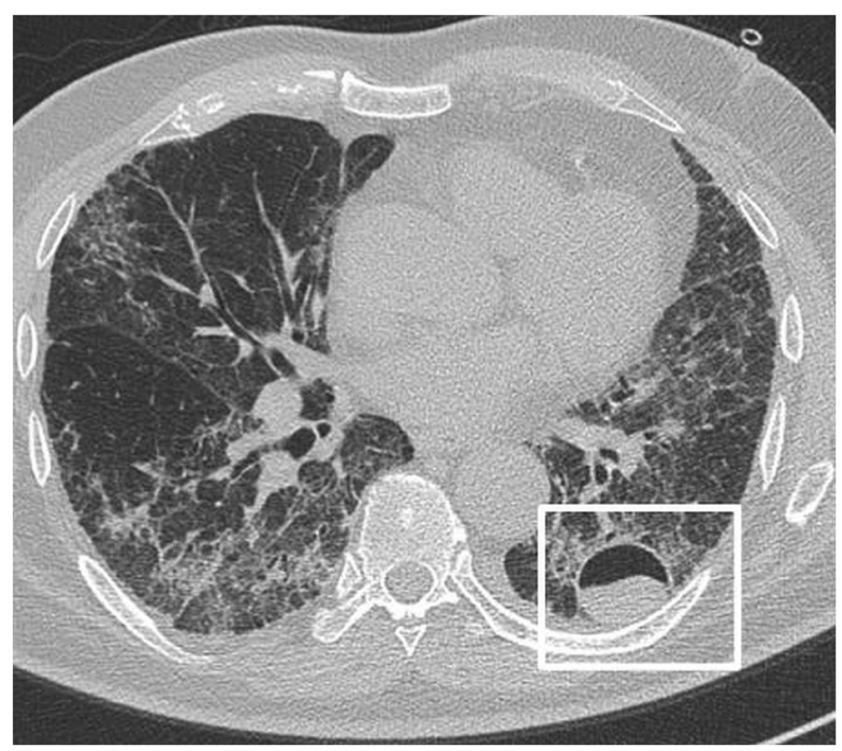

Fig. 12 Chest CT of a 73-year-old COVID-19 patient, showing a bilateral diffuse reticular pattern of abnormalities; in the left lower lobe, posteriorly, cavitation is visible as an air-filled component including a fluid level (frame) 


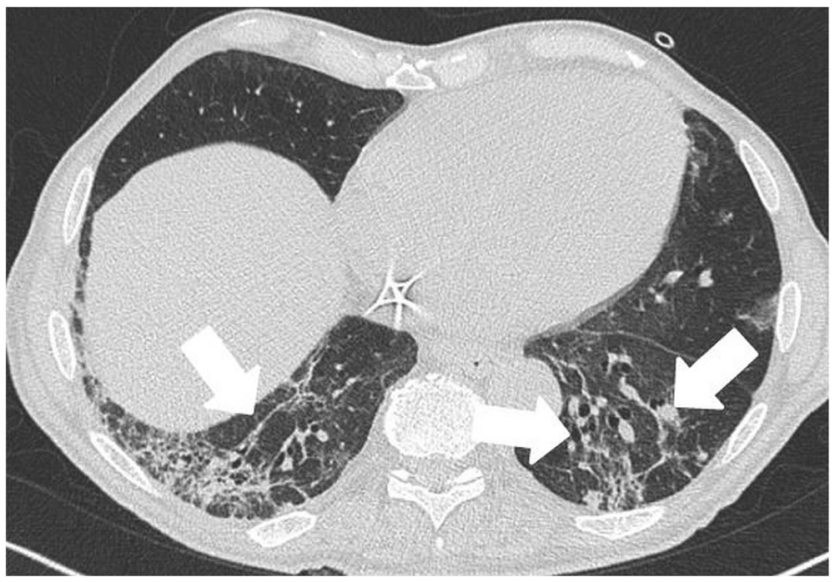

Fig. 13 Pre-discharge chest CT of a COVID-19 male patient, at 27 days from the onset of the symptoms. Bilateral linear opacities are recognizable (white arrows)

In fact, the CT execution for large cohorts of patients carries multiple risks, as the depletion of personal protective equipment, due to excessive usage, transport of critical patients, increased risk of viral transmission, ionizing radiation exposures, and the time required to clean and disinfect equipment after imaging of patients suspected of having COVID-19 [22].

CT findings of COVID-19 infection are nonspecific, highly overlapping with those of other types of viral pneumonia []. Different imaging features and patterns have been described, as well as different time course and disease severity [232425].

The most common findings are GGO, consolidation, and crazy paving appearance, with typical bilateral, peripheral, and basal in distribution [23-25]. Different imaging manifestations can be related to different examinations timing after the onset of the patients' symptoms, but maybe also correlated to different pre-existing patients' clinical characteristics/comorbidities.
GGO are defined as hazy areas of slightly increased density without cancelation of bronchial and vascular contours that may be caused by the partial displacement of air due to partial filling of airspaces or interstitial thickening [26]; in COVID19 patients, they may be unilateral or bilateral, mainly with a subpleural distribution, and have been reported with an incidence up to $91 \%$ [25, 27] (Fig. 6).

Consolidation consists of alveolar air replaced by pathological fluids, cells, or tissue, on chest CT is visible as increased pulmonary parenchymal density that obscures the margins of the underlying vessels and airway walls (Fig. 7). In COVID-19, consolidation is reported with variable frequency, from 5\% [28] to $63 \%$ [25], with increased frequency at 2 weeks after symptoms onset [29].

Crazy paving has been observed in up to $36 \%$ of cases [30] and is represented by thickened interlobular septa and intralobular lines superimposed on a GGO background; this sign may result from the alveolar edema and interstitial inflammatory of acute lung injury [31] (Fig. 8).

The reticular pattern is the thickening of pulmonary interstitial structures such as interlobular septa and intralobular lines [26] and was reported in up to 22\% of patients [32] (Fig. 9).

Air bronchogram is recognizable as air-filled bronchi on a background of the opaque lung (Fig. 8); the reported incidence in COVID-19 patients is up to $80 \%$ [32]. Bronchial wall thickening is considered a manifestation of bronchial wall inflammatory damage and has been found in up to $23 \%$ of cases [30].

Halo sign is represented by nodules or masses surrounded by GGO and described with an incidence of $2.4 \%$ [33].

Subpleural linear opacities are curvilinear opacities parallel to the pleural surface observed in $20 \%$ of cases [30] (Fig. 10.)

The air bubble sign is a small air containing space; it might be a pathological dilatation of a physiological space or a crosssection of bronchiectasis or could be associated with the process of consolidation resorption [30] (Fig. 11).

Fig. 14 Coronal reconstruction of the pre- (a) and post-discharge CT a 75-year-old COVID-19 patient. The first CT (a), executed on April 15, at 30 days from the onset of the symptoms, showed bilateral irregular linear opacities and peripheral consolidations. The follow-up CT, performed on June 24 , showed bilateral extensive faded GGO in the location of the previous abnormalities. This can be defined as a "tinted sign"
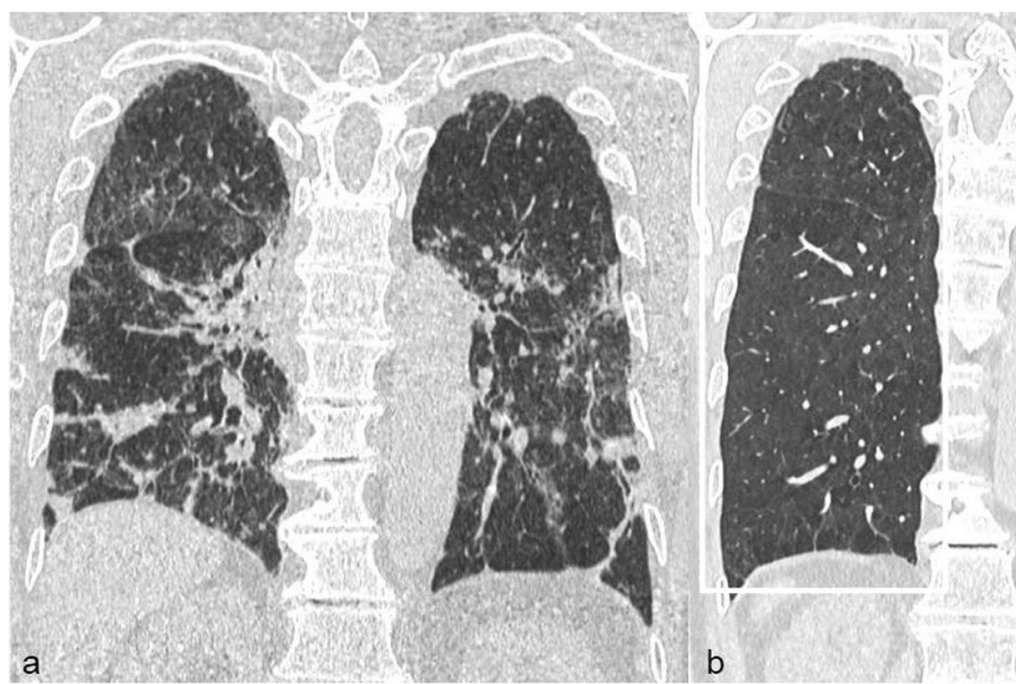
The vascular enlargement is the dilatation of the pulmonary vessels around or within the airspace opacities, reported in about $64 \%$ of cases [33].

Mediastinal lymphadenopathies are lymph nodes with short axis $>1 \mathrm{~cm}$; together with pleural effusion are considered risk factors for severe disease [30]. Their mean incidence is about $5 \%$ [33].

Cavitation consists of a gas-filled space, delimited by thick walls that can contain a fluid level. Its origin is uncertain: It can result from the cystic degeneration of lung consolidation or can be considered a sign of superinfection; the incidence is $0.1 \%$ [33] (Fig. 12).

Chest abnormalities in COVID-19 pneumonia change over time. GGO tend to progress in extent and attenuation value and evolve either towards crazy paving areas or towards linear and retractile consolidation areas. Lung alterations are maximal at around day 10-12 after the onset of the symptoms and then generally decrease progressively in size and attenuation value. Four stages have been reported: early/initial stage ( 0 4 days from the onset of the symptoms), normal CT or GGO only; progressive stage (5-8 days), increased GGO and crazy paving appearance; peak stage (9-13 days), predominance of consolidation; and absorption stage (>14 days), improvement in the lung abnormalities with the appearance of the fibrous stripes (Fig. 13) [24].

In discharged patients, the "tinted sign," visible as an extension of the GGO with a decreased density, has been observed with an incidence of 32\% (Fig. 14) [34].

\section{Conclusion}

In conclusion, we illustrated the imaging findings of COVID19 pneumonia that can be assessed with all the available imaging techniques. Appropriate knowledge of these features helps radiologists and clinicians in the diagnosis and management of the affected patients.

Author's contributions M Cellina: Conceptualization; Data curation; Writing - original draft

C Martinenghi: Data curation; Supervision; Validation

P Marino: Investigation; Resources

G Oliva: Writing - review and editing

\section{Compliance with ethical standards}

Conflict of interest The authors declare that they have no conflict of interest.

\section{References}

1. Zhu N, Zhang D, Wang W, Li X, Yang B, Song J, Zhao X, Huang B, Shi W, Lu R, Niu P, Zhan F, Ma X, Wang D, Xu W, Wu G, Gao
GF, Tan W (2020) China novel coronavirus investigating and research team. A novel coronavirus from patients with pneumonia in China, 2019. N Engl J Med20;382(8):727-733. https://doi.org/10. 1056/NEJMoa2001017

2. Coronaviridae Study Group of the International Committee on Taxonomy of Viruses (2020) The species Severe acute respiratory syndrome-related coronavirus: classifying 2019-nCoV and naming it SARS-CoV-2. Nat Microbiol 5(4):536-544. https://doi.org/10. 1038/s41564-020-0695-Z

3. WHO Coronavirus disease (COVID-19) dashboard. https:// covid19.who.int/ accessed November 22

4. Guan WJ, Ni ZY, Hu Y, Liang WH, Ou CQ, He JX, Liu L, Shan H, Lei CL, Hui DSC, Du B, Li LJ, Zeng G, Yuen KY, Chen RC, Tang CL, Wang T, Chen PY, Xiang J, Li SY, Wang JL, Liang ZJ, Peng YX, Wei L, Liu Y, Hu YH, Peng P, Wang JM, Liu JY, Chen Z, Li G, Zheng ZJ, Qiu SQ, Luo J, Ye CJ, Zhu SY, Zhong NS (2020) China medical treatment expert group for Covid-19. Clinical characteristics of coronavirus disease 2019 in China. N Engl J med 30;382(18):1708-1720. https://doi.org/10.1056/NEJMoa2002032

5. Hu Z, Song C, Xu C, Jin G, Chen Y, Xu X, Ma H, Chen W, Lin Y, Zheng Y, Wang J, Hu Z, Yi Y, Shen H (2020) Clinical characteristics of 24 asymptomatic infections with COVID-19 screened among close contacts in Nanjing, China. Sci China Life Sci 63(5): 706-711. https://doi.org/10.1007/s11427-020-1661-4

6. Vernuccio F, Giambelluca D, Cannella R, Lombardo FP, Panzuto F, Midiri M, Cabassa P (2020) Radiographic and chest CT imaging presentation and follow-up of COVID-19 pneumonia: a multicenter experience from an endemic area. Emerg Radiol 11:1-10. https:// doi.org/10.1007/s10140-020-01817-x

7. Rubin GD, Ryerson CJ, Haramati LB, Sverzellati N, Kanne JP, Raoof S, Schluger NW, Volpi A, Yim JJ, Martin IBK, Anderson DJ, Kong C, Altes T, Bush A, Desai SR, Goldin O, Goo JM, Humbert M, Inoue Y, Kauczor HU, Luo F, Mazzone PJ, Prokop M, Remy-Jardin M, Richeldi L, Schaefer-Prokop CM, Tomiyama N, Wells AU, Leung AN (2020) The role of chest imaging in patient management during the COVID-19 pandemic: a multinational consensus statement from the Fleischner society. Radiology 296(1):172-180. https://doi.org/10.1148/radiol.2020201365

8. Pakray A, Walker D, Figacz A, Kilanowski S, Rhodes C, Doshi S, Coffey M (2020) Imaging evaluation of COVID-19 in the emergency department. Emerg Radiol 27(6):579-588. https://doi.org/ 10.1007/s10140-020-01787-0

9. Diagnosis and treatment protocols of COVID-19 infection (trial version 5). The National Health Commission of the People's Republic of China. http://www.nhc.gov.cn/yzygj/ s7653p/202002/ 3b09b894ac9b4204a79db5b8912d4440.shtml. Accessed 4 Feb 2020

10. Sofia S, Boccatonda A, Montanari M, Spampinato M, D'ardes D, Cocco G, Accogli E, Cipollone F, Schiavone C (2020) Thoracic ultrasound and SARS-COVID-19: a pictorial essay. J Ultrasound 23(2):217-221. https://doi.org/10.1007/s40477-020-00458-7

11. Allinovi M, Parise A, Giacalone M, Amerio A, Delsante M, Odone A, Franci A, Gigliotti F, Amadasi S, Delmonte D, Parri N, Mangia A (2020) Lung ultrasound may support diagnosis and monitoring of COVID-19 pneumonia. Ultrasound Med Biol 46(11):2908-2917. https://doi.org/10.1016/j.ultrasmedbio.2020.07.018

12. Wong HYF, Lam HYS, Fong AH, Leung ST, Chin TW, Lo CSY, Lui MM, Lee JCY, Chiu KW, Chung TW, Lee EYP, Wan EYF, Hung IFN, Lam TPW, Kuo MD, Ng MY (2020) Frequency and distribution of chest radiographic findings in patients positive for COVID-19. Radiology 296(2):E72-E78. https://doi.org/10.1148/ radiol.2020201160

13. ACR Recommendations for the use of chest radiography and computed tomography (CT) for suspected COVID-19 infection | American College of Radiology. https://www.acr.org/Advocacyand-Economics/ACR-Position-Statements/Recommendations-for- 
Chest-Radiography-and-CT-for-Suspected-COVID19-Infection, accessed March 11, 2020

14. Orsi MA, Oliva G, Toluian T, Valenti Pittino C, Panzeri M, Cellina M (2020) Feasibility, reproducibility, and clinical validity of a quantitative chest X-ray assessment for COVID-19. Am J Trop Med Hyg 103(2):822-827. https://doi.org/10.4269/ajtmh.20-0535

15. Rodrigues JCL, Hare SS, Edey A, Devaraj A, Jacob J, Johnstone A, McStay R, Nair A, Robinson G (2020) An update on COVID-19 for the radiologist - a British society of thoracic imaging statement. Clin Radiol 75(5):323-325. https://doi.org/10.1016/j.crad.2020.03. 003

16. Cellina M, Panzeri M, Oliva G (2020) Chest radiograph features predict a favorable outcome in patients with COVID-19. Radiology 297(1):E238. https://doi.org/10.1148/radiol.2020202326

17. Kim HW, Capaccione KM, Li G, Luk L, Widemon RS, Rahman O, Beylergil V, Mitchell R, D'Souza BM, Leb JS, Dumeer S, BentleyHibbert S, Liu M, Jambawalikar S, Austin JHM, Salvatore M (2020) The role of initial chest X-ray in triaging patients with suspected COVID-19 during the pandemic. Emerg Radiol 27(6): 617-621. https://doi.org/10.1007/s10140-020-01808-y

18. Li K, Wu J, Wu F, Guo D, Chen L, Fang Z, Li C (2020) The clinical and chest CT features associated with severe and critical COVID19 pneumonia. Investig Radiol 55(6):327-331. https://doi.org/10. 1097/RLI.0000000000000672

19. Selvaraj V, Dapaah-Afriyie K (2020) Lung cavitation due to COVID-19 pneumonia. BMJ Case Rep 13(7):e237245. https:// doi.org/10.1136/bcr-2020-237245

20. Salehi S, Abedi A, Balakrishnan S, Gholamrezanezhad A (2020) Coronavirus disease 2019 (COVID-19): a systematic review of imaging findings in 919 patients. AJR Am J Roentgenol 215(1):8793. https://doi.org/10.2214/AJR.20.23034

21. López Vega JM, Parra Gordo ML, Diez Tascón A, Ossaba Vélez S (2020) Pneumomediastinum and spontaneous pneumothorax as an extrapulmonary complication of COVID-19 disease. Emerg Radiol 27(6):727-730. https://doi.org/10.1007/s10140-020-01806-0

22. Cellina M, Orsi M, Oliva G (2020) How to reorganize the radiology departments to face the 2019 coronavirus disease outbreak. Disaster med public health prep 1-3. https://doi.org/10.1017/dmp.2020.159

23. Barbosa CS, Chaves GWOG, de Oliveira CV, Bachion GH, Chi CK, Cerri GG, Lima TC, Lee HJ (2020) COVID-19 pneumonia in the emergency department: correlation of initial chest CT findings with short-term outcome. Emerg Radiol 27(6):691-699. https://doi. org/10.1007/s10140-020-01863-5

24. Pan F, Ye T, Sun P, Gui S, Liang B, Li L, Zheng D, Wang J, Hesketh RL, Yang L, Zheng (2020) Time course of lung changes at chest CT during recovery from coronavirus disease 2019 (COVID-19). Radiology 295(3):715-721. https://doi.org/10.1148/ radiol.2020200370

25. Wu J, Wu X, Zeng W, Guo D, Fang Z, Chen L, Huang H, Li C Chest CT findings in patients with coronavirus disease 2019 And its relationship with clinical features. Investig Radiol 55(5):257-261. https://doi.org/10.1097/RLI.0000000000000670

26. Hansell DM, Bankier AA, MacMahon H, McLoud TC, Müller NL, Remy J (2008) Fleischner society: glossary of terms for thoracic imaging. Radiology 246(3):697-722. https://doi.org/10.1148/ radiol.2462070712

27. Majidi H, Bani-Mostafavi ES, Mardanshahi Z, Godazandeh F, Ghasemian R, Heydari K, Alizadeh-Navaei R (2020) Highresolution computed tomography finding in 552 patients with symptomatic COVID-19: first report from north of Iran. Emerg Radiol 27(6):633-639. https://doi.org/10.1007/s10140-02001819-9

28. Shi H, Han X, Jiang N, Cao Y, Alwalid O, Gu J, Fan Y, Zheng C (2020) Radiological findings from 81 patients with COVID-19 pneumonia in Wuhan, China: a descriptive study. Lancet Infect Dis 20(4):425-434. https://doi.org/10.1016/S1473-3099(20) 30086-4

29. Pan Y, Guan H, Zhou S, Wang Y, Li Q, Zhu T, Hu Q, Xia L (2020) Initial CT findings and temporal changes in patients with the novel coronavirus pneumonia (2019-nCoV): a study of 63 patients in Wuhan, China. Eur Radiol 30(6):3306-3309. https://doi.org/10. 1007/s00330-020-06731-x

30. Colombi D, Villani GD, Maffi G, Risoli C, Bodini FC, Petrini M, Morelli N, Anselmi P, Milanese G, Silva M, Sverzellati N, Michieletti E. Qualitative and quantitative chest CT parameters as predictors of specific mortality in COVID-19 patients. Emerg Radiol 27(6):701-710. https://doi.org/10.1007/s10140-02001867-1

31. Ye Z, Zhang Y, Wang Y, Huang Z, Song B (2020) Chest CT manifestations of new coronavirus disease 2019 (COVID-19): a pictorial review. Eur Radiol 30(8):4381-4389. https://doi.org/10. 1007/s00330-020-06801-0

32. Song F, Shi N, Shan F, Zhang Z, Shen J, Lu H, Ling Y, Jiang Y, Shi Y (2020) Emerging 2019 novel coronavirus (2019-nCoV) pneumonia. Radiology 295(1):210-217. https://doi.org/10.1148/radiol. 2020200274

33. Ojha V, Mani A, Pandey NN, Sharma S, Kumar S (2020) CT in coronavirus disease 2019 (COVID-19): a systematic review of chest CT findings in 4410 adult patients. Eur Radiol 30:1-10. https://doi.org/10.1007/s00330-020-06975-7

34. Liu D, Zhang W, Pan F, Li L, Yang L, Zheng D, Wang J, Liang B (2020) The pulmonary sequalae in discharged patients with COVID-19: a short-term observational study. Respir res 24;21(1): 125. https://doi.org/10.1186/s12931-020-01385-1

Publisher's note Springer Nature remains neutral with regard to jurisdictional claims in published maps and institutional affiliations. 